\title{
Rho Kinase Inhibition as a Therapeutic for Progressive Supranuclear Palsy and Corticobasal Degeneration
}

\author{
Erik G. Gentry, ${ }^{1,2}$ Benjamin W. Henderson, ${ }^{1,2}$ Andrew E. Arrant,, ${ }^{1,2}$ Marla Gearing, ${ }^{5}$ Yangbo Feng, ${ }^{6}{ }^{-N i c o l e ~ C . ~ R i d d l e, ~}{ }^{4}$ \\ and Jeremy $\mathrm{H}$. Herskowitz ${ }^{1,2,3}$ \\ ${ }^{1}$ Center for Neurodegeneration and Experimental Therapeutics and Departments of ${ }^{2}$ Neurology, ${ }^{3}$ Neurobiology, and ${ }^{4}$ Biology, University of Alabama at \\ Birmingham, Birmingham, Alabama 35294, ${ }^{5}$ Departments of Pathology and Laboratory Medicine, and Neurology, Emory University School of Medicine, \\ Atlanta, Georgia 30322, and ${ }^{6}$ Medicinal Chemistry, Translational Research Institute, Scripps Research Institute, Jupiter, Florida 33458
}

Progressive supranuclear palsy (PSP) and corticobasal degeneration (CBD) are neurodegenerative four-repeat tauopathies with no cure. Mitigating pathogenic tau levels is a rational strategy for tauopathy treatment, but therapeutic targets with clinically available drugs are lacking. Here, we report that protein levels of the Rho-associated protein kinases (ROCK1 and ROCK2), p70 S6 kinase (S6K), and mammalian target of rapamycin (mTOR) were increased in PSP and CBD brains. RNAi depletion of ROCK1 or ROCK2 reduced tau mRNA and protein level in human neuroblastoma cells. However, additional phenotypes were observed under ROCK2 knockdown, including decreased S6K and phosphorylated mTOR levels. Pharmacologic inhibition of Rho kinases in neurons diminished detergent-soluble and -insoluble tau through a combination of autophagy enhancement and tau mRNA reduction. Fasudil, a clinically approved ROCK inhibitor, suppressed rough eye phenotype and mitigated pathogenic tau levels by inducing autophagic pathways in a Drosophila model of tauopathy. Collectively, these findings highlight the Rho kinases as rational therapeutic targets to combat tau accumulation in PSP and CBD.

Key words: autophagy; Drosophila; Rho kinase; ROCK; tau; tauopathy

Significance Statement

Studies of progressive supranuclear palsy (PSP) and corticobasal degeneration (CBD) suggest that mitigating pathogenic tau levels is a rational strategy for tauopathy treatment. In this report, the Rho-associated protein kinases (ROCK1 and ROCK2) are identified as novel drug targets for PSP and CBD. We show that elevated insoluble tau levels are associated with increased ROCK1 and ROCK2 in PSP and CBD brains, whereas experiments in cellular and animal models identify pharmacologic inhibition of ROCKs as a mechanism-based approach to reduce tau levels. Our study correlates bona fide changes in PSP and CBD brains with cellular models, identifies drug targets, and tests the therapeutic in vivo.

\section{Introduction}

Progressive supranuclear palsy (PSP) and corticobasal degeneration (CBD) are progressive neurodegenerative four-repeat (4R) tauopathies with disease onset in the fifth to seventh decades of

\footnotetext{
Received June 18, 2015; revised Dec. 14, 2015; accepted Dec. 18, 2015.

Author contributions: A.E.A., N.C.R., and J.H.H. designed research; E.G.G., B.W.H., A.E.A., N.C.R., and J.H.H. performed research; M.G. and Y.F. contributed unpublished reagents/analytic tools; E.G.G., B.W.H., A.E.A., Y.F., N.C.R., and J.H.H. analyzed data; J.H.H. wrote the paper.

This work was supported by National Institutes of Health/National Institute on Aging Grant 5R00AG043552-04 (J.H.H.), National Institute of Neurological Disorders and Stroke Neuroscience Core Facilities Grant NS055077, Alzheimer's Disease Research Center Grant AG025688 at Emory University, and Alzheimer's Association New Investigator Research Grant 2015-NIRG-339422 (J.H.H.).

The authors declare no competing financial interests.

Correspondence should be addressed to Dr. Jeremy H. Herskowitz, Center for Neurodegeneration and Experimental Therapeutics, Departments of Neurology and Neurobiology, University of Alabama at Birmingham, 1825 University Bouldvard, Birmingham, AL 35294. E-mail: jhersko@uab.edu.

DOI:10.1523/JNEUROSCI.2336-15.2016

Copyright $\odot 2016$ the authors $\quad 0270-6474 / 16 / 361316-08 \$ 15.00 / 0$
}

life (Kouri et al., 2011). PSP and CBD are neuropathologically characterized by preferential accumulation of $4 \mathrm{R}$ tau isoform in neurons, astrocytes, and oligodendroglia, and both diseases share genetic features, including a higher frequency of the microtubule associated protein tau (MAPT) H1 haplotype (Conrad et al., 1997; Baker et al., 1999; Houlden et al., 2001).

The hypothesis that accumulation of tau oligomers or fibrils is toxic to neurons and other brain cells suggests that enhancing cellular catabolic pathways would be a beneficial strategy to combat PSP, CBD, and/or other tauopathies. Autophagy (macroautophagy) mediates protein aggregate degradation and represents the major cellular pathway for soluble tau disposal in primary neuron cultures (Verhoef et al., 2002; Krüger et al., 2012). Autophagy can be pharmacologically induced with rapamycin through the inhibition of mammalian target of rapamycin (mTOR), and treatment with rapamycin reduced insoluble tau levels in Drosophila models of tauopathy (Berger et al., 2006; 
Bakhoum et al., 2014). Other studies revealed that $\mathrm{mTOR}$ and the mTOR effector p70 S6 kinase (S6K) likely have direct effects on tau synthesis, phosphorylation, and aggregation (An et al., 2003; Tang et al., 2013).

Rho-associated coiled-coil containing protein kinases 1 (ROCK1) and ROCK2 are ubiquitous serine/threonine kinases that share $65 \%$ similarity in their amino acid sequences and $92 \%$ identity in their kinase domains (Nakagawa et al., 1996). Pharmacologic inhibition of ROCKs can induce protein degradation pathways, including autophagy, in mammalian cells (Bauer et al., 2009; Koch et al., 2014). However, whether ROCKs influence tau synthesis or protein homeostasis is unclear. In this study, observations from PSP and CBD brains are linked to in vitro and in vivo models that provide mechanistic insight, identifying ROCKs as therapeutic targets for PSP and CBD.

\section{Materials and Methods}

Antibodies. The following antibodies were used: Tau Dako A0024; phospho-Tau (S202) CP13 Cell Signaling Technology 11834; phosphoTau (S396) PHF13 Cell Signaling Technology 9632; ROCK1 Abcam ab45171; ROCK2 Abcam ab56661; mTOR Cell Signaling Technology 2972s; phospho-mTOR (S2448) Cell Signaling Technology 2971s; phospho-S6K (T389) Cell Signaling Technology 108D2; S6K Cell Signaling Technology 2708; p62 Abcam ab109012; LC3 Novus NB100-2220; Actin Abcam ab6276.

Human brain tissue preparation and immunoblotting. Postmortem frontal cortex tissues from control, PSP, and CBD cases were selected for comparison from the Emory University Alzheimer's Disease Research Center brain bank. The PSP and CBD cases in this study underwent extensive neuropathological characterization required for diagnosis based on established criteria (Hauw et al., 1994; Cairns et al., 2007). Cases were matched as closely as possible for age at death, gender, and postmortem interval (Table 1). Sarkosyl-soluble and -insoluble fractions were prepared exactly as described previously (Diner et al., 2014). Protein concentration was determined by bicinchoninic acid method (Pierce). Immunoblotting was performed using standard procedures as described previously (Herskowitz et al., 2011). Fifty micrograms of protein per sample were used for immunoblots. Actin was used as loading control. Images were captured using an Odyssey Image Station (Li-Cor), and band intensities were quantified using Odyssey Application Software version 3.0 ( $\mathrm{Li}$-Cor).

For densitometry analysis, control human brain samples were divided randomly into two groups (1-5 and 6-10). Control samples $1-5$ were run on an immunoblot with all PSP cases and a separate immunoblot with all CBD cases. In parallel, control samples 6-10 were run on an immunoblot with all PSP cases and a separate immunoblot with all CBD cases. For each immunoblot, densitometry measurements for the control samples were averaged and normalized to an arbitrary unit of 1 . Because each control case was used on two immunoblots, two densitometry measurements were acquired for each control case. The two densitometry measurements for each control sample were averaged, and that average represents the single data point that is represented in the densitometry graphs in Figure 1.

Cell culture, transduction, and transfection. SH-SY5Y human neuroblastoma cells were maintained in MEM Eagle (Lonza) with 10\% fetal bovine serum and $1 \%$ penicillin/streptomycin. For transductions or transfections, equivalent amounts of cells were plated. DNA plasmid or siRNA transfections were performed using Lipofectamine 2000 (Invitrogen) or DharmaFECT 1 siRNA Transfection Reagent (Dharmacon), respectively, according to the instructions of the manufacturer. Primary cortical neuronal cultures were prepared from embryonic day 17 C57BL/6 mouse embryos and maintained in Neurobasal medium supplemented with $0.8 \mathrm{~mm}$ L-glutamine and B27 as described previously (Herskowitz et al., 2013).

DNA constructs, lentivirus, and siRNA. Lentivirus vectors for shRNA expression were constructed and generated as described previously (Herskowitz et al., 2012): ROCK1 shRNA1, 5'-GCCAATGACTTACTTAGGA
Table 1. Postmortem human brain tissue samples

\begin{tabular}{|c|c|c|c|c|}
\hline & PMI (h) & Age at onset & Age at death & Race/sex \\
\hline \multicolumn{5}{|c|}{ Case control $(n=10)$} \\
\hline 1 & 6 & & 58 & WM \\
\hline 2 & 8 & & 60 & $\mathrm{AF}$ \\
\hline 3 & 65 & & WM & \\
\hline 4 & 10 & & 66 & WM \\
\hline 5 & 4.5 & & 70 & HM \\
\hline 6 & 3 & & 74 & WF \\
\hline 7 & 6.5 & & 46 & WF \\
\hline 8 & 6.5 & & 53 & AM \\
\hline 9 & 17 & & 57 & $\mathrm{AF}$ \\
\hline 10 & 10 & & 57 & WM \\
\hline \multicolumn{5}{|c|}{$\operatorname{PSP}(n=9)$} \\
\hline 1 & 15 & 61 & 70 & WF \\
\hline 2 & 11.5 & 74 & 82 & WF \\
\hline 3 & 23 & 77 & 83 & WM \\
\hline 4 & 10 & 65 & 75 & WM \\
\hline 5 & 14 & 89 & 94 & WF \\
\hline 6 & 10 & 70 & 83 & WM \\
\hline 7 & 6 & 65 & 71 & WF \\
\hline 8 & 3 & 51 & 61 & WM \\
\hline 9 & 10 & 70 & 83 & WM \\
\hline \multicolumn{5}{|c|}{$\mathrm{CBD}(n=9)$} \\
\hline 1 & 24 & 91 & M & \\
\hline 2 & 8.5 & 72 & 78 & WF \\
\hline 3 & 5 & 57 & 62 & WM \\
\hline 4 & 2.75 & 56 & 61 & HM \\
\hline 5 & 24 & 33 & 44 & WM \\
\hline 6 & $<8$ & 53 & 65 & WM \\
\hline 7 & 3 & 72 & 82 & WF \\
\hline 8 & 3 & 63 & 68 & WF \\
\hline 9 & 4 & 78 & 86 & WF \\
\hline
\end{tabular}

Case numbers correspond to immunoblot samples in Figure 1. If values are blank, information was unavailable. PMI, Postmortem interval. Race: $W$, white; $A$, African descent; $H$, Hispanic; Sex: $F$, female; $M$, male.

and shRNA2, 5' -CTACAAGTGTTGCTAGTTT; ROCK2 shRNA1, 5' ATCAGACAGCATCCTTTCT and shRNA2, 5'-GCAAATCTGTTAA TACTCG; and scramble, 5' -GGACTACTCTAGACGTATA. ROCK2 mutants resistant to shRNA1 sequences were produced as described previously (Herskowitz et al., 2013). To generate ROCK2 K121G, cDNA encoding human ROCK2 with shRNA-resistant mutations was used as a template, and the QuikChange XL Site-Directed Mutagenesis kit (Stratagene) was used: sense primer, 5'-GGCATCGCAGAAGGTTTATGCTATGGGGCTTCT TAGTAAGTTTGA, and antisense primer, $5^{\prime}$-TCAAACTTACTAAGA AGCCCCATAGCATAAACCTTCTGCGATGCC. Constructs were verified by sequencing. For S6K, the following were used: siRNA1, 5'-CAUGGAAC AUUGUGAGAAA and siRNA2, 5'-GGAAUGGGCAUAAGUUGUA.

Chemicals. To inhibit ROCKs, Fasudil (HA-1077; Sigma-Aldrich) or SR3677 (N-[2-[2-(dimethylamino)ethoxy]-4-(1 H-pyrazol-4-yl)phenyl2,3-dihydro-1,4-benzodioxin-2-carboxamide dihydrochloride; Feng et al., 2008) was dissolved in $\mathrm{H}_{2} \mathrm{O}$, and mock was $\mathrm{H}_{2} \mathrm{O}$. MG132 (carbobenzoxy-Lleucyl-L-leucyl-L-leucinal), bafilomycin, and rapamycin (Sigma catalog \#C2211, \#B1793, and \#R0395, respectively) were dissolved in 100\% dimethylsulfoxide (DMSO) and used at $10 \mu \mathrm{M}, 100 \mathrm{nM}$, or $5 \mu \mathrm{M}$, respectively. Mock was DMSO.

Cell lysate preparation and viability assay. Cells were lysed in PBS plus protease inhibitor mixture (Roche Diagnostics), Halt phosphatase inhibitor mixture (Pierce), and lysis buffer containing $0.5 \%$ Nonidet P-40, $0.5 \%$ deoxycholate, $150 \mathrm{~mm}$ sodium chloride, and $50 \mathrm{~mm}$ Tris, $\mathrm{pH}$ 7.4. Cell lysate was subjected to a 13,000 rpm spin, and the cleared lysate was designated as the soluble fraction. Sarkosyl-insoluble fractions were prepared as described above for human brain tissue analysis; however, a Dounce homogenizer was not used (Diner et al., 2014). Protein concentration was determined for all fractions as described above. Cell viability was measured using the CellTiter 96 Non-Radioactive Cell Proliferation Assay (Promega) following the instructions of the manufacturer. Absor- 


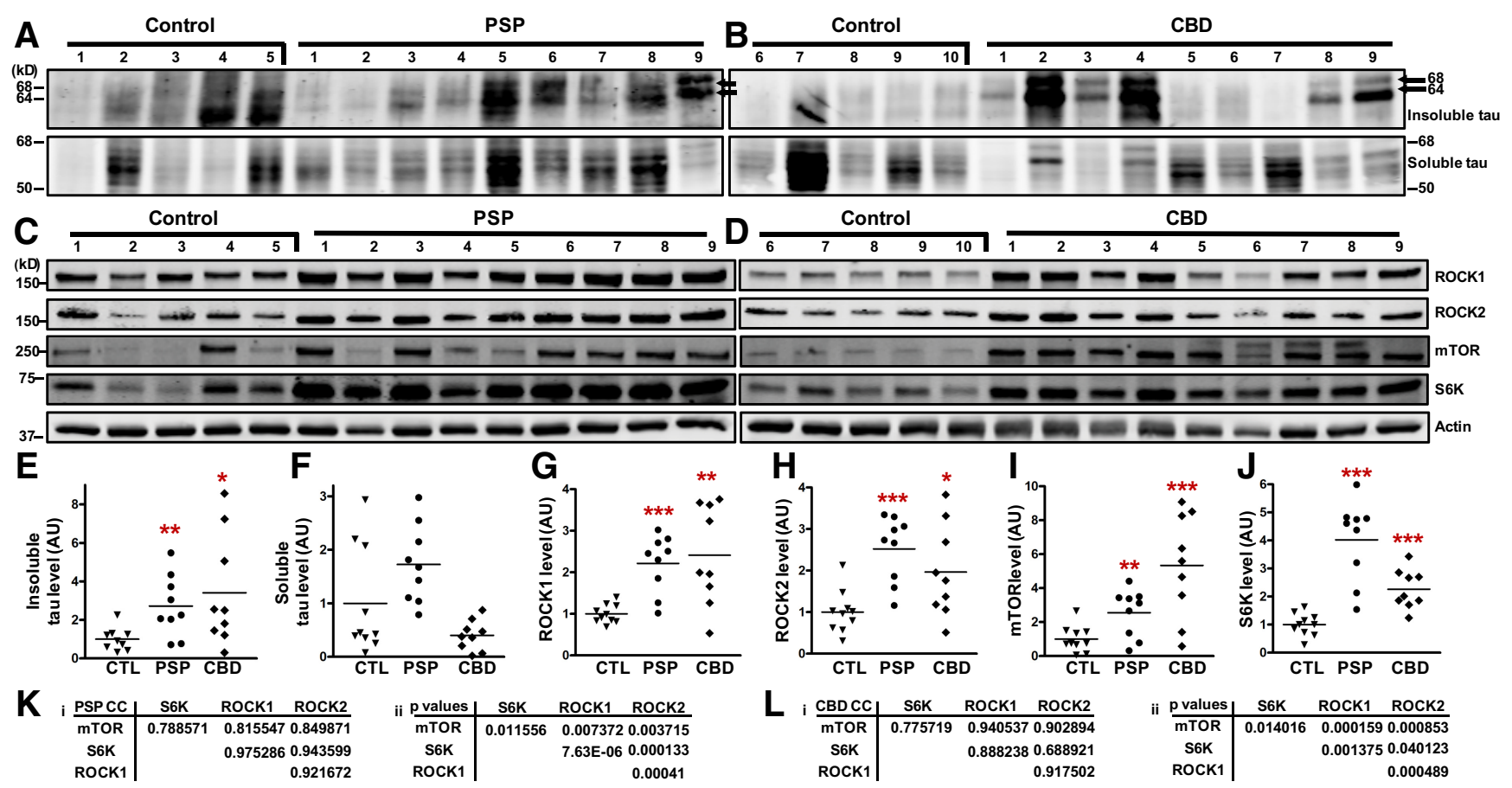

Figure 1. Increased ROCKs, $m$ TOR, and S6K in PSP and CBD brains. $A-D$, Representative immunoblots from control (CTL), PSP, or CBD brains. E-J, Densitometry analysis of immunoblots. Each case is expressed as an individual data point, and lines represent the mean. $\boldsymbol{E}^{*} p=0.0258,{ }^{* *} p=0.0080 . \boldsymbol{G},{ }^{* *} p=0.0018,{ }^{* * *} p \leq 0.0001 . \boldsymbol{H}^{*}{ }^{*} p=0.0235,{ }^{*{ }^{*}} p=0.0001 . \boldsymbol{I}$, ${ }^{* *} p=0.0073,{ }^{* * *} p=$ $0.0004 . J,{ }^{* * *} p<0.0001 . K, L$, Correlation coefficients (i) and $p$ values (ii) for PSP and CBD cases. AU, Arbitrary units.

bance was measured at $570 \mathrm{~nm}$ with a reference wavelength at $650 \mathrm{~nm}$. Cell death values were calculated as the percentage of control absorbance.

$q R T-P C R$. RNA was prepared with TRIzol reagent (Life Technologies 15596-026) following the instructions of the manufacturer. cDNA was generated with the SuperScript III First Strand Synthesis System (Life Technologies 18080-051). cDNA was quantified using LightCycler 480 Probes master mix (Roche) and TaqMan Gene Expression Assays for human Tau (Hs00902194_m1), GAPDH (Hs02758991_g1), rodent MAPT (Rn00691532_m1), and $\beta$-actin (Mm00607939_s1). Amplification was performed on a Roche LightCycler 480. Data was quantified using the $\Delta \Delta$ Ct method (User Bulletin 2; Applied Biosystems).

Drosophila studies. Flies expressing human tau were obtained from the Bloomington Drosophila Stock Center (stock 51360). They were maintained at $25^{\circ} \mathrm{C}$ and $70 \%$ humidity on Jazzmix media (Thermo Fisher Scientific). For drug treatments, flies were grown on Formula 4-24 Instant Medium (Carolina Biological Supply) prepared with water (mock) or indicated drug doses. Eye phenotypes were examined using a S8APO dissecting microscope equipped with a ring light (Leica), and images were captured using a DFC295 camera (Leica) with Leica software. Eye images were analyzed in NIH ImageJ using published protocols (Costa et al., 2011). Fly head homogenates were prepared as described above for soluble fraction cell lysate.

Statistical analysis. Statistical analysis was performed using Student's $t$ test for independent samples. Data are expressed as the percentage of the mean \pm SEM with respect to the control. Error bars represent SEM. Prism software (GraphPad Software) was used for correlation analysis and the generation of all graphs, correlation, and $p$ value matrices.

\section{Results}

To measure tau levels in the cases under study, a one-step fractionation protocol was used to generate Sarkosyl-soluble and -insoluble fractions from 10 control, nine PSP, and nine CBD postmortem cortical tissue homogenates (Table 1). All fractions were subjected to SDS-PAGE and subsequent immunoblot using polyclonal tau antibody generated against amino acids 243-441, containing microtubule-binding 4R (Fig. 1 $A, B$ ). In Sarkosylinsoluble PSP and CBD fractions, tau immunoreactivity was observed prominently at 68 and $64 \mathrm{kDa}$, representing hyperphosphorylated full-length $4 \mathrm{R}$ tau isoforms (Liu et al., 2001). Densitometry analysis indicated that Sarkosyl-insoluble tau level was increased significantly in PSP and CBD cases compared with control brains (Fig. 1E). To quantify Sarkosyl-soluble tau species, immunoreactive bands from 50 to $68 \mathrm{kDa}$ were measured, but changes in soluble tau level were not statistically significant among control, PSP, and CBD samples (Fig. $1 F$ ).

Tau degradation can be mediated by autophagy, and mTOR signaling through S6K can initiate autophagic cascades in neurons (Boland et al., 2008; Krüger et al., 2012). Moreover, ROCK1 and/or ROCK2 are proposed to influence autophagy in mammalian cells (Bauer et al., 2009). To assess whether protein levels of these kinases change in PSP or CBD, Sarkosyl-soluble samples were subjected to SDS-PAGE and subsequent immunoblot (Fig. $1 C, D)$. Densitometry analysis indicated that ROCK1, ROCK2, $\mathrm{mTOR}$, and S6K are increased significantly in PSP and CBD cases compared with controls (Fig. $1 G-J$ ). Statistical correlation analysis was performed, and correlation coefficients and $p$ values indicate strong positive correlation among ROCK1, ROCK2, mTOR, and S6K in PSP and CBD cases (Fig. $1 K, L$ ).

Next, we sought to determine how these kinases may affect tau levels in cellular models. First, SH-SY5Y human neuroblastoma cells were transduced with lentivirus expressing ROCK1-targeted, ROCK2-targeted, or scramble shRNA (two shRNA sequences per target), and $96 \mathrm{~h}$ later, levels of endogenous tau protein and tau mRNA were measured by immunoblot or quantitative PCR, respectively. Tau protein was reduced 34 and $65 \%$, whereas tau mRNA was reduced $\sim 51$ and $\sim 61 \%$ in ROCK 1 or ROCK 2 knockdown cells, respectively, suggesting that lower tau mRNA levels may contribute to tau protein loss (Fig. 2A,B).

Phosphorylation of mTOR at serine 2448 (pmTOR) suppresses autophagy induction, and pmTOR level can be regulated by phosphatidylinositol 3-kinase signaling through AKT or via 

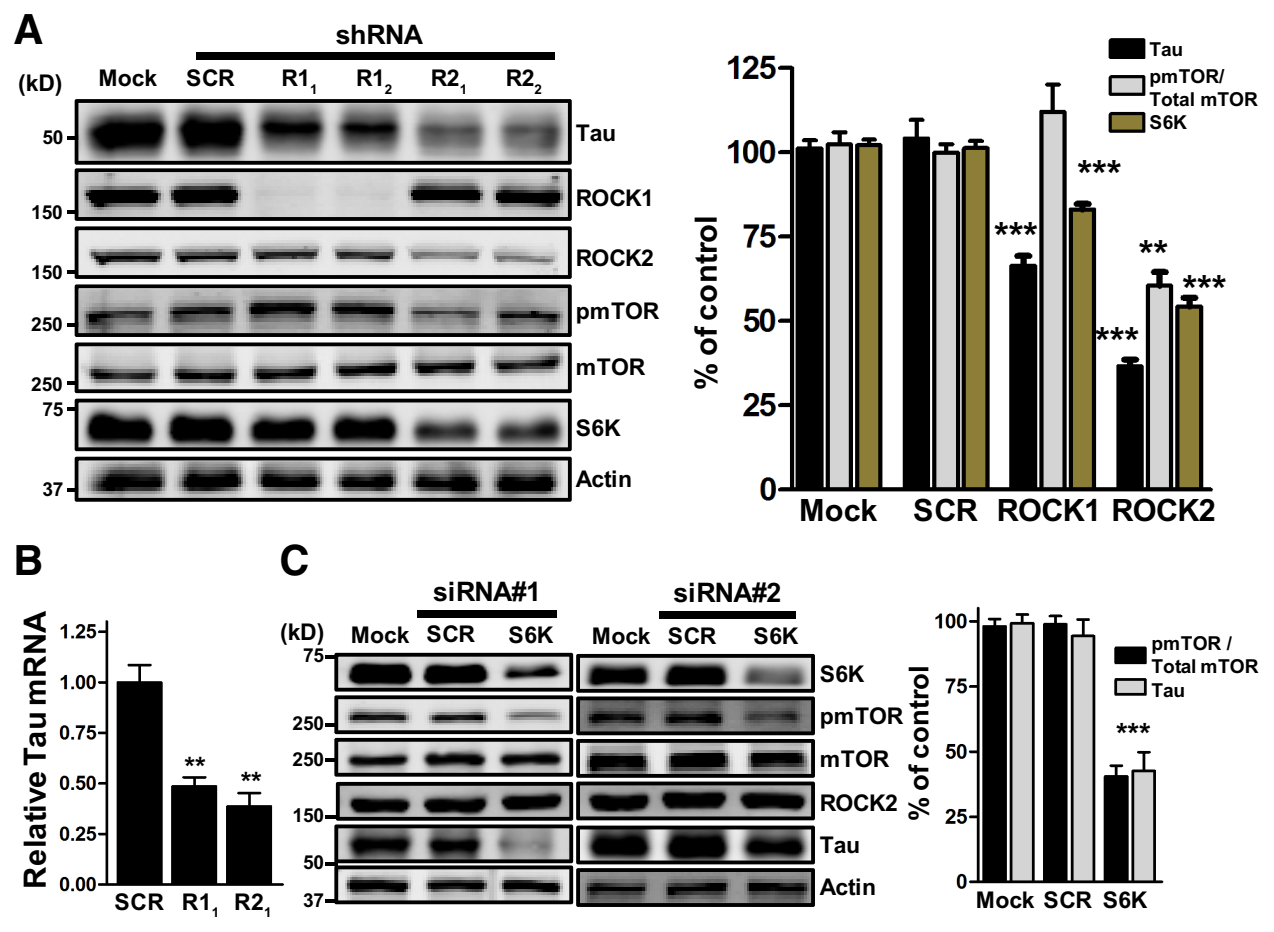

C
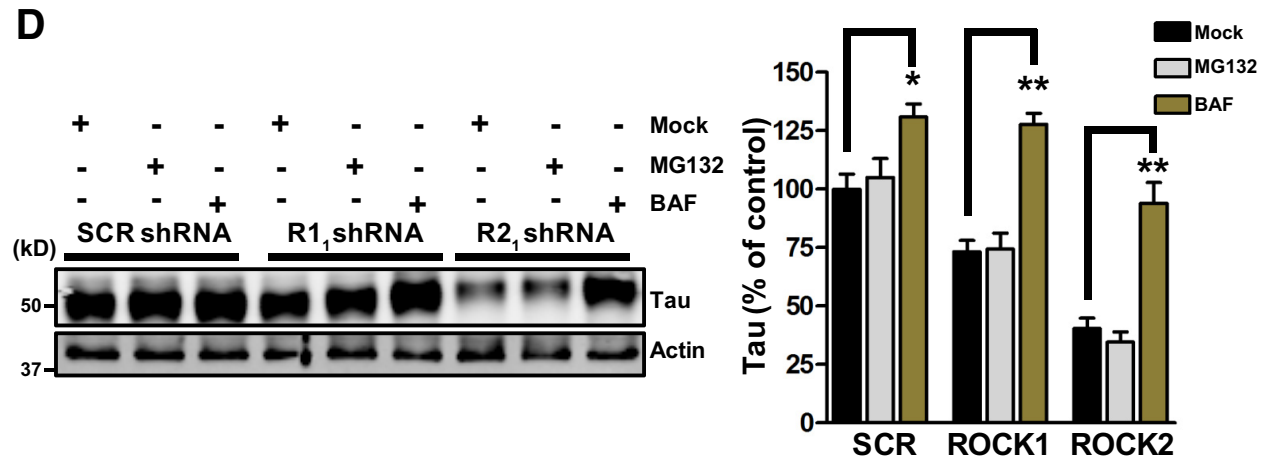

E
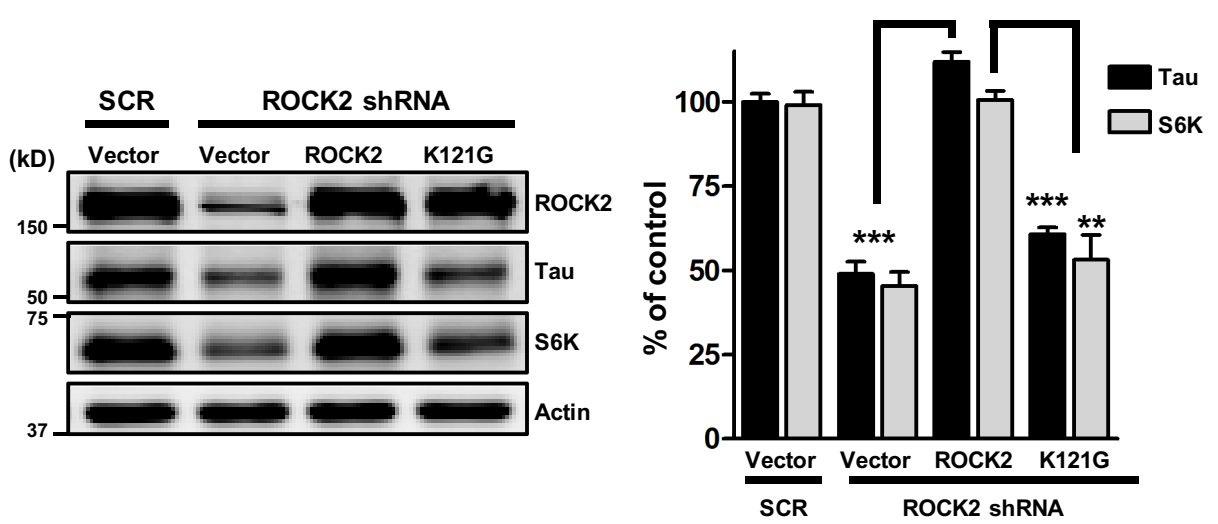

Figure 2. ROCK2 kinase activity is required for the effects of ROCK2 on tau. Representative immunoblots of kinase knockdown compared with mock or scramble (SCR) controls. Unless otherwise

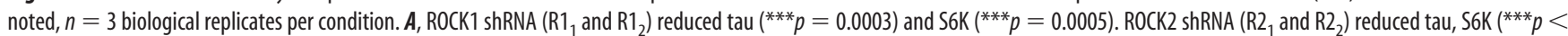
0.0001 ), and pMTOR (normalized to total mTOR; $\left.\left.{ }^{* *} p=0.0016\right) \cdot \boldsymbol{B}, \mathrm{R} 1_{1}{ }^{* *} p=0.005\right)$ and $\mathrm{R}_{2}\left({ }^{* *} p=0.0026\right)$ reduced relative tau mRNA level. $n=6$ biological replicates per condition. $\boldsymbol{C}$, S6K knockdown reduced pmTOR and tau ( $\left.{ }^{* * *} p<0.0001\right)$. D , Tau accumulates in bafilomycin-treated samples. $S C R,{ }^{*} p=0.0228 ; R 1_{1},{ }^{* *} p=0.0013 ; R 2_{1},{ }^{* *} p=0.0057$. E, ROCK2 expression reversed S6K and tau levels compared with R2 1 plus vector control $\left({ }^{* * *} p=0.0002,{ }^{* * *} p=0.0004\right)$ or L121G $\left({ }^{* *} p=0.0039,{ }^{* * *} p=0.0001\right)$. All data are expressed as the percentage of the mean \pm SEM.

direct phosphorylation by S6K (Chiang and Abraham, 2005; Holz and Blenis, 2005). Densitometry analysis indicated that S6K was decreased 17 and $45 \%$ after RNAi depletion of ROCK1 or ROCK2, respectively, and that ROCK2, but not ROCK1, knockdown decreased pmTOR, whereas total mTOR levels were un- changed (Fig. 2A). Based on this, we hypothesized that depletion of ROCK2 reduced S6K levels, which in turn suppressed pmTOR, stimulating autophagy and tau degradation. To test the first half of the hypothesis, SH-SY5Y cells were transfected with S6Ktargeted or scramble siRNA, and $96 \mathrm{~h}$ later, tau levels and pmTOR 

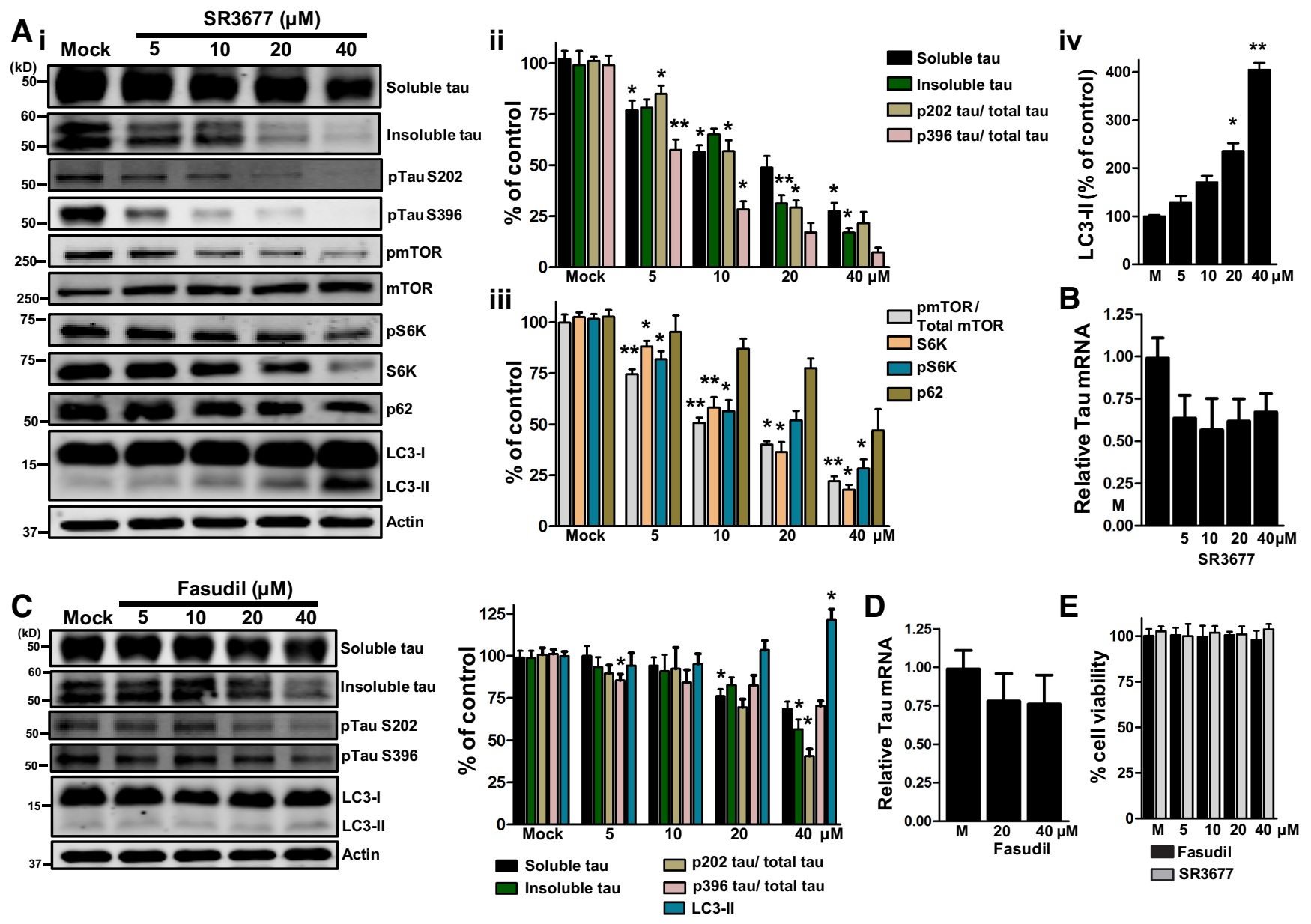

Figure 3. Pharmacologic inhibition of ROCKs reduces tau in neurons. Unless otherwise noted, $n=3$ biological replicates per condition. Measurements of tau and mTOR phosphorylation were normalized to overall tau and mTOR levels, respectively. Statistics data for $\boldsymbol{A}, \boldsymbol{i i}$-iv, and $\mathbf{C}$ are located in Table 2 . Ai, Representative blots are shown. Aii, Densitometry analysis of soluble tau, insoluble tau, and tau phosphorylation. Aiii, Densitometry analysis of autophagy-related markers after SR3677 treatment. Aiv, Densitometry analysis of LC3-II after SR3677 treatment. B, SR3677 reduced tau mRNA level $(5 \mu \mathrm{M}, 36 \% ; 10 \mu \mathrm{M}, 43 \% ; 20 \mu \mathrm{m}, 38 \% ; 40 \mu \mathrm{M}, 32 \%) . n=6$ biological replicates per condition. C, Representative blots are shown. Densitometry analysis of soluble tau, insoluble tau, tau phosphorylation, and LC3-II levels after Fasudil treatment. D, Fasudil reduced tau mRNA level $(20 \mu \mathrm{m}, 22 \% ; 40 \mu \mathrm{m}, 24 \%) . n=6$ biological replicates per condition. $\boldsymbol{E}$, No gross changes in cell viability were observed at the indicated doses of Fasudil or SR3677 treatments. All data are expressed as the percentage of the mean \pm SEM with respect to mock.

were measured by immunoblot (Fig. 2C). Densitometry analysis indicated that tau and pmTOR were reduced 57 and $60 \%$, respectively, in S6K-depleted cells. Notably, ROCK2 levels were unchanged after S6K knockdown (Fig. 2C). These results suggest that ROCK2 knockdown reduces $56 \mathrm{~K}$ levels and in turn decreases pmTOR and tau. Next, ROCK1-targeted, ROCK2-targeted, or scramble shRNA-expressing cells were treated for $24 \mathrm{~h}$ with MG132, a proteasome inhibitor, or $6 \mathrm{~h}$ with bafilomycin, an endosomal acidification inhibitor that blocks autophagy, and tau levels were measured by immunoblot. MG132 treatment did not alter tau levels, whereas bafilomycin increased tau significantly in all samples (Fig. 2D). These observations indicate that inhibiting autophagy, but not the proteasome, accumulates tau protein in ROCK1 or ROCK2 knockdown cells.

To determine whether ROCK2 kinase activity mediates the effects of ROCK2 on S6K and tau, site-directed mutagenesis was used to substitute glycine for lysine 121, generating the ROCK2 mutant K121G. Lysine 121 is the ATP-binding site in the kinase domain and is absolutely required for ROCK2 activity (Amano et al., 1997). SH-SY5Y cells were transduced with lentivirus expressing ROCK2-targeted or scramble shRNA, and $72 \mathrm{~h}$ later, cells were transiently transfected with the indicated ROCK2 plasmids (resistant to shRNA) or empty vector control. In ROCK2- depleted cells, expression of ROCK2 reversed effects on S6K and tau, whereas K121G did not (Fig. 2E). These results demonstrate that ROCK2 kinase activity is required for the effects of ROCK2 on S6K and tau.

To test whether pharmacologic inhibition of ROCK2 stimulates autophagy and reduces tau levels, primary murine cortical neurons were exposed to SR3677, a characterized ROCK2 small-molecule inhibitor (Feng et al., 2008; Herskowitz et al., 2013). After $16 \mathrm{~h}$, endogenous proteins were measured by immunoblot (Fig. 3A). Densitometry analysis indicated that SR3677 treatment showed a linear dose-dependent decrease in soluble tau, $\mathrm{S} 6 \mathrm{~K}$, and pmTOR protein levels, which were consistent with ROCK2 knockdown studies (Fig. 3A). Suppressing mTOR phosphorylation of S6K threonine 389 (pS6K) induces autophagy in neurons; therefore, pS6K was measured, as well as LC3-II, a specific marker for autophagosomes, and p62, a known substrate of autophagy (Kabeya et al., 2000; Biørkøy et al., 2005; Boland et al., 2008). SR3677 treatment increased LC3-II, whereas p62 and pS6K levels were decreased in a dose-dependent manner (Fig. 3A). Sarkosyl-insoluble fractions were also prepared, and tau was measured by immunoblot. Densitometry analysis indicated that SR3677 treatment showed a linear dose-dependent decrease in Sar- 
Table 2. Immunoblot measurements and statistics for SR3677 and Fasudil treatments

\begin{tabular}{|c|c|c|c|c|c|c|c|c|c|}
\hline Compound & Soluble tau & Insoluble tau & p202 tau/tau & p396 tau/tau & LC3-II & pmTOR/mTOR & S6K & pS6K & p62 \\
\hline \multicolumn{10}{|l|}{ SR3677 } \\
\hline \multicolumn{10}{|l|}{ Mock } \\
\hline$\%$ & 102.1 & 99.06 & 101.2 & 99.12 & 99.78 & 99.74 & 102.5 & 101.6 & 102.7 \\
\hline \multicolumn{10}{|l|}{$5 \mu \mathrm{M}$} \\
\hline$\%$ & 77.05 & 78.22 & 84.94 & 57.48 & 127.6 & 74.47 & 88.12 & 81.82 & 95.17 \\
\hline Mock vs $5 \mu \mathrm{M}$ & ${ }^{*} p=0.0144$ & $p=0.061$ & ${ }^{*} p=0.0234$ & ${ }^{* *} p=0.0039$ & $p=0.1333$ & ${ }^{* *} p=0.006$ & ${ }^{*} p=0.0169$ & ${ }^{*} p=0.0129$ & $p=0.4411$ \\
\hline \multicolumn{10}{|l|}{$10 \mu \mathrm{M}$} \\
\hline$\%$ & 56.48 & 65.13 & 56.88 & 28.39 & 170.7 & 50.78 & 58.2 & 56.46 & 87.01 \\
\hline 5 vs $10 \mu \mathrm{m}$ & ${ }^{*} p=0.0220$ & $p=0.0574$ & ${ }^{*} p=0.0144$ & ${ }^{*} p=0.0111$ & $p=0.0938$ & ${ }^{* *} p=0.0026$ & ${ }^{* *} p=0.0069$ & ${ }^{*} p=0.0192$ & $p=0.4378$ \\
\hline \multicolumn{10}{|l|}{$20 \mu \mathrm{M}$} \\
\hline$\%$ & 48.82 & 31.21 & 29.24 & 17.03 & 235.5 & 40.12 & 36.37 & 51.9 & 77.48 \\
\hline 10 vs $20 \mu \mathrm{m}$ & $p=0.3122$ & ${ }^{* *} p=0.0024$ & ${ }^{*} p=0.0125$ & $p=0.1343$ & ${ }^{*} p=0.0369$ & ${ }^{*} p=0.0249$ & ${ }^{*} p=0.0388$ & $p=0.5571$ & $p=0.2329$ \\
\hline \multicolumn{10}{|l|}{$40 \mu \mathrm{m}$} \\
\hline$\%$ & 27.37 & 16.91 & 21.49 & 7.267 & 404.5 & 22.08 & 17.89 & 28.28 & 47.04 \\
\hline 20 vs $40 \mu \mathrm{m}$ & ${ }^{*} p=0.0378$ & ${ }^{*} p=0.0356$ & $p=0.3055$ & $p=0.1319$ & ${ }^{* *} p=0.0015$ & ${ }^{* *} p=0.0031$ & ${ }^{*} p=0.0297$ & ${ }^{*} p=0.0228$ & $p=0.0561$ \\
\hline \multicolumn{10}{|l|}{ Fasudil } \\
\hline \multicolumn{10}{|l|}{ Mock } \\
\hline$\%$ & 98.91 & 98.58 & 100.7 & 101.1 & 99.78 & & & & \\
\hline \multicolumn{10}{|l|}{$5 \mu \mathrm{M}$} \\
\hline$\%$ & 99.96 & 93.34 & 89.54 & 85.42 & 94.21 & & & & \\
\hline Mock vs $5 \mu \mathrm{M}$ & $p=0.889$ & $p=0.5146$ & $p=0.1534$ & ${ }^{*} p=0.0268$ & $p=0.5225$ & & & & \\
\hline \multicolumn{10}{|l|}{$10 \mu \mathrm{m}$} \\
\hline$\%$ & 94.16 & 90.79 & 92.41 & 84.17 & 95.13 & & & & \\
\hline 5 vs $10 \mu \mathrm{m}$ & $p=0.4891$ & $p=0.8351$ & $p=0.8415$ & $p=0.8888$ & $p=0.9285$ & & & & \\
\hline \multicolumn{10}{|l|}{$20 \mu \mathrm{M}$} \\
\hline$\%$ & 76.11 & 82.74 & 69.54 & 82.51 & 103.4 & & & & \\
\hline 10 vs $20 \mu \mathrm{M}$ & ${ }^{*} p=0.049$ & $p=0.5014$ & $p=0.1629$ & $p=0.8698$ & $p=0.3743$ & & & & \\
\hline \multicolumn{10}{|l|}{$40 \mu \mathrm{m}$} \\
\hline$\%$ & 68.54 & 56.45 & 40.66 & 70.28 & 121.2 & & & & \\
\hline 20 vs $40 \mu \mathrm{M}$ & $p=0.2758$ & ${ }^{*} p=0.024$ & ${ }^{*} p=0.0104$ & $p=0.1406$ & ${ }^{*} p=0.0368 \#$ & & & & \\
\hline
\end{tabular}

Measurements and statistics data for Figure $3, A$ and C. Percentage values represent the percentage of the mean with respect to mock. $p$ values are provided for the indicated comparison. ${ }^{\#}$ Mock versus $40 \mu \mathrm{m}$.

kosyl-insoluble tau levels that mimicked SR3677-mediated effects on soluble tau (Fig. $3 A$ ). Notably, tau immunoreactivity was observed prominently at two bands in Sarkosyl-insoluble fractions, which may represent phosphorylated tau isoforms.

Past studies demonstrated that pharmacologic inhibition of ROCKs can suppress phosphorylation of tau at serine 202 (pTau S202) and serine 396 (pTau S396; Castro-Alvarez et al., 2011; Hamano et al., 2012). To determine whether SR3677 reduces endogenous pTau S202 or pTau S396 in primary neurons, immunoblots were performed. Levels of pTau S202 and pTau S396 were normalized to soluble tau, and densitometry analysis indicated that exposure to SR3677 led to a linear dose-dependent decrease in pTau S202 and pTau S396 (Fig. 3A).

Quantitative PCR analysis revealed that tau mRNA levels were lower but not significantly reduced after SR3677 treatments compared with mock (Fig. 3B). Autophagy can mediate protein aggregate degradation (Verhoef et al., 2002). Based on this and the findings presented here, we hypothesize that ROCK2 inhibition stimulates autophagy and that this mechanism in combination with lower tau mRNA levels contributes to soluble and insoluble tau protein reduction in neurons.

To assess the clinical relevance of our hypothesis, cortical neurons were exposed for $16 \mathrm{~h}$ to increasing doses of Fasudil, a clinically approved ROCK inhibitor (Shibuya et al., 2005). Densitometry analysis indicated that soluble tau protein level was significantly reduced at doses $\geq 20 \mu \mathrm{M}$, whereas insoluble tau and pTau S202 was decreased significantly at $40 \mu \mathrm{M}$ (Fig. $3 C)$. Notably, LC3-II was increased significantly at $40 \mu \mathrm{M}$ compared with mock (Fig. 3C). Quantitative PCR analysis showed lower, but not significantly reduced, tau mRNA levels in neurons treated with Fasudil $\geq 20 \mu \mathrm{M}$ (Fig. 3D). Notably, cell viability of primary neuronal cultures was not affected by Fasudil or SR3677 treatment (Fig. 3E). Detailed statistical information for densitometry analyses of SR3677 and Fasudil treatments is presented in Table 2 .

To determine whether Fasudil can suppress pathogenic tau in vivo, a Drosophila model of tauopathy was used. In this model, the glass multiple reporter element drives expression of a single-copy cDNA encoding the $4 \mathrm{R}$ human wild-type tau in the eye (referred to as gl-tau). gl-tau eyes display a rough phenotype in anterior regions, and large autophagic vacuoles accumulate in the retina, suggesting that autophagy is impaired (Jackson et al., 2002; Bakhoum et al., 2014). Drosophila express a single isoform of ROCK (DROCK), and amino acid sequence alignments of DROCK with rodent ROCKs indicate a mismatch that is adjacent to the ATP binding site in the DROCK kinase domain (Mizuno et al., 1999). Based on this, Fasudil was selected to pharmacologically suppress DROCK. Rapamycin induces autophagic pathways and reduces the rough eye phenotype in gl-tau flies (Bakhoum et al., 2014). Therefore, rapamycin was used as a positive control in our experiments. gl-tau flies were grown in the presence or absence of $30 \mu \mathrm{M}$ Fasudil or $5 \mu \mathrm{M}$ rapamycin, and immediately after eclosion, adult fly heads were harvested for microscopy and biochemical analyses. The rough eye phenotype was similarly alleviated in Fasudil- and rapamycin-treated cohorts compared with mock (Fig. $4 A$ ). In parallel, immunoblot and subsequent densitometry analysis indicated that $4 \mathrm{R}$ human tau protein level was reduced 62 and $64 \%$ in flies exposed to Fasudil and rapamycin, respectively (Fig. 4B). Additional bio- 
chemical evaluation indicated that rapamycin treatment decreased levels of Ref(2)P, the Drosophila homolog of mammalian p62, and suppressed phosphorylation of Drosophila target of rapamycin (pTOR) in gl-tau flies (Miron et al., 2003; Nezis et al., 2008). Ref(2)P and pTOR were similarly reduced in cohorts exposed to Fasudil (Fig. 4B). Based on these findings, we propose that Fasudil induces autophagic pathways that mitigate pathogenic tau levels in gl-tau flies.

\section{Discussion}

Mitigating pathogenic tau levels is a rational strategy for tauopathy treatment, but therapeutic targets with clinically available drugs are lacking. Currently, 29 small-molecule protein kinase inhibitors are used for the treatment of human diseases. Of these, Fasudil and Ripasudil are ROCK inhibitors. Our findings indicate that ROCK1 and ROCK2 are elevated in PSP and CBD brains and that shRNAmediated depletion or pharmacologic inhibition of ROCKs reduces tau through combinatorial mechanisms at the mRNA and protein levels.

Whether increased ROCK1, ROCK2, S6K, and/or mTOR contribute to underlying pathogenic mechanisms or manifest during PSP and CBD progression is a critical question. Analysis of PSP and CBD brains at earlier disease stages or complimentary experiments in tauopathy mouse models may help address these questions. It is possible that increased kinase levels may be attributable in part to reactive gliosis in PSP and/or CBD brains (Togo and Dickson, 2002). Hence, it will be important to determine whether changes to ROCKs, S6K, or mTOR are exclusive to specific cell populations and whether enhanced gene transcription, inefficient protein degradation, or a combination of these effects could elevate kinases at the cellular level.

In human neuroblastoma cells, ROCK1 knockdown lowered tau mRNA $\sim 51 \%$ but reduced tau protein only $34 \%$ (Fig. $2 A, B$ ). This disconnect may reflect lengthy tau protein half-life, which is $\sim 20$ h in SH-SY5Y cells (Kim et al., 2014). Therefore, the effects of lower tau mRNA may require another $20 \mathrm{~h}$ or more before tau protein effects are appreciable by immunoblot. To extrapolate this further, ROCK2 knockdown decreased tau mRNA $\sim 61 \%$ and reduced tau protein $65 \%$, suggesting that posttranslational mechanisms may also play a role in tau protein loss. Blocking autophagy, but not the proteasome, rescued tau protein level, and coupled with decreased pmTOR, we propose that autophagy stimulation facilitates tau protein degradation in ROCK2depleted cells. Similarly, pharmacologic inhibition of ROCK2 using SR3677 in neurons lowered tau mRNA levels equivalently at all doses, whereas tau protein level was depleted in a dosedependent manner (Fig. $3 A, B$ ). Diminished tau protein levels in SR3677-treated neurons were accompanied by protein changes that hallmark autophagy induction, including decreases in pmTOR, pS6K, and p62, as well as increases in LC3-II. These findings support our hypothesis that ROCK2 inhibition promotes autophagic degradation of tau, but how shRNA-mediated deple- tion or pharmacologic inhibition of ROCKs lowers tau mRNA level is an open question and will require additional investigation. Notably, tau inclusions are found in neurons, astrocytes, and oligodendroglia in PSP and CBD brains, and although strong evidence supports the hypothesis that tau degradation is mediated by autophagy in neurons, it is possible that other mechanisms, including proteasome-mediated degradation, are critical for tau disposal in non-neuronal cells (Wang and Mandelkow, 2012).

Compared with Fasudil, which inhibits ROCK1 and ROCK2 with similar potency, SR3677 offers approximately eightfold higher selectivity of ROCK2 over ROCK1 (Feng et al., 2008). This may account for the more robust SR3677induced phenotypes and similarity to ROCK2 knockdown studies, yet it is likely that SR3677 also inhibits ROCK1 at the experimental doses used in this study. Moreover, at high doses $(40 \mu \mathrm{M})$ of SR3677, it is possible that off-target effects, such as direct inhibition of $\mathrm{mTOR}$, could contribute to the observed phenotypes. The off-target hit rate of SR3677 was $1.4 \%$, and the cell-based $\mathrm{IC}_{50}$ for A7r5 smooth muscle cells was calculated to be $\sim 3$ nM. However, SR3677 potency in cell culture experiments is likely affected by cell accumulation, cell permeability, and/or ROCK1 and ROCK2 enzyme kinetics (Feng et al., 2008). We propose that suppressing ROCK1 activity likely has therapeutic benefit, but tau reduction is more robustly induced by ROCK2 inhibition through combinatorial effects on tau mRNA levels and autophagy stimulation. Coupled with the clinical availability of Fasudil, our findings raise enthusiasm for ROCK inhibitors as potential therapeutics to combat tau accumulation in PSP and CBD. 


\section{References}

Amano M, Chihara K, Kimura K, Fukata Y, Nakamura N, Matsuura Y, Kaibuchi K (1997) Formation of actin stress fibers and focal adhesions enhanced by Rho-kinase. Science 275:1308-1311. CrossRef Medline

An WL, Cowburn RF, Li L, Braak H, Alafuzoff I, Iqbal K, Iqbal IG, Winblad B, Pei JJ (2003) Up-regulation of phosphorylated/activated p70 S6 kinase and its relationship to neurofibrillary pathology in Alzheimer's disease. Am J Pathol 163:591-607. CrossRef Medline

Baker M, Litvan I, Houlden H, Adamson J, Dickson D, Perez-Tur J, Hardy J, Lynch T, Bigio E, Hutton M (1999) Association of an extended haplotype in the tau gene with progressive supranuclear palsy. Hum Mol Genet 8:711-715. CrossRef Medline

Bakhoum MF, Bakhoum CY, Ding Z, Carlton SM, Campbell GA, Jackson GR (2014) Evidence for autophagic gridlock in aging and neurodegeneration. Transl Res 164:1-12. CrossRef Medline

Bauer PO, Wong HK, Oyama F, Goswami A, Okuno M, Kino Y, Miyazaki H, Nukina N (2009) Inhibition of Rho kinases enhances the degradation of mutant huntingtin. J Biol Chem 284:13153-13164. CrossRef Medline

Berger Z, Ravikumar B, Menzies FM, Oroz LG, Underwood BR, Pangalos MN, Schmitt I, Wullner U, Evert BO, O'Kane CJ, Rubinsztein DC (2006) Rapamycin alleviates toxicity of different aggregate-prone proteins. Hum Mol Genet 15:433-442. CrossRef Medline

Bjørkøy G, Lamark T, Brech A, Outzen H, Perander M, Overvatn A, Stenmark $\mathrm{H}$, Johansen T (2005) p62/SQSTM1 forms protein aggregates degraded by autophagy and has a protective effect on huntingtin-induced cell death. J Cell Biol 171:603-614. CrossRef Medline

Boland B, Kumar A, Lee S, Platt FM, Wegiel J, Yu WH, Nixon RA (2008) Autophagy induction and autophagosome clearance in neurons: relationship to autophagic pathology in Alzheimer's disease. J Neurosci 28: 6926-6937. CrossRef Medline

Cairns NJ, Bigio EH, Mackenzie IR, Neumann M, Lee VM, Hatanpaa KJ, White CL 3rd, Schneider JA, Grinberg LT, Halliday G, Duyckaerts C, Lowe JS, Holm IE, Tolnay M, Okamoto K, Yokoo H, Murayama S, Woulfe J, Munoz DG, Dickson DW, Ince PG, Trojanowski JQ, Mann DM (2007) Neuropathologic diagnostic and nosologic criteria for frontotemporal lobar degeneration: consensus of the Consortium for Frontotemporal Lobar Degeneration. Acta Neuropathol 114:5-22. CrossRef Medline

Castro-Alvarez JF, Gutierrez-Vargas J, Darnaudéry M, Cardona-Gómez GP (2011) ROCK inhibition prevents tau hyperphosphorylation and p25/ CDK5 increase after global cerebral ischemia. Behav Neurosci 125: 465-472. CrossRef Medline

Chiang GG, Abraham RT (2005) Phosphorylation of mammalian target of rapamycin (mTOR) at Ser-2448 is mediated by p70S6 kinase. J Biol Chem 280:25485-25490. CrossRef Medline

Conrad C, Andreadis A, Trojanowski JQ, Dickson DW, Kang D, Chen X, Wiederholt W, Hansen L, Masliah E, Thal LJ, Katzman R, Xia Y, Saitoh T (1997) Genetic evidence for the involvement of tau in progressive supranuclear palsy. Ann Neurol 41:277-281. CrossRef Medline

Costa R, Speretta E, Crowther DC, Cardoso I (2011) Testing the therapeutic potential of doxycycline in a Drosophila melanogaster model of Alzheimer disease. J Biol Chem 286:41647-41655. CrossRef Medline

Diner I, Hales CM, Bishof I, Rabenold L, Duong DM, Yi H, Laur O, Gearing M, Troncoso J, Thambisetty M, Lah JJ, Levey AI, Seyfried NT (2014) Aggregation properties of the small nuclear ribonucleoprotein U1-70K in Alzheimer disease. J Biol Chem 289:35296-35313. CrossRef Medline

Feng Y, Yin Y, Weiser A, Griffin E, Cameron MD, Lin L, Ruiz C, Schürer SC, Inoue T, Rao PV, Schröter T, Lograsso P (2008) Discovery of substituted 4-(pyrazol-4-yl)-phenylbenzodioxane-2-carboxamides as potent and highly selective Rho kinase (ROCK-II) inhibitors. J Med Chem 51: 6642-6645. CrossRef Medline

Hamano T, Yen SH, Gendron T, Ko LW, Kuriyama M (2012) Pitavastatin decreases tau levels via the inactivation of Rho/ROCK. Neurobiol Aging 33:2306-2320. CrossRef Medline

Hauw JJ, Daniel SE, Dickson D, Horoupian DS, Jellinger K, Lantos PL, McKee A, Tabaton M, Litvan I (1994) Preliminary NINDS neuropathologic criteria for Steele-Richardson-Olszewski syndrome (progressive supranuclear palsy). Neurology 44:2015-2019. CrossRef Medline

Herskowitz JH, Seyfried NT, Gearing M, Kahn RA, Peng J, Levey AI, Lah JJ (2011) Rho kinase II phosphorylation of the lipoprotein receptor LR11/ SORLA alters amyloid-beta production. J Biol Chem 286:6117-6127. CrossRef Medline

Herskowitz JH, Offe K, Deshpande A, Kahn RA, Levey AI, Lah JJ (2012)
GGA1-mediated endocytic traffic of LR11/SorLA alters APP intracellular distribution and amyloid-beta production. Mol Biol Cell 23:2645-2657. CrossRef Medline

Herskowitz JH, Feng Y, Mattheyses AL, Hales CM, Higginbotham LA, Duong DM, Montine TJ, Troncoso JC, Thambisetty M, Seyfried NT, Levey AI, Lah JJ (2013) Pharmacologic inhibition of ROCK2 suppresses amyloidbeta production in an Alzheimer's disease mouse model. J Neurosci 33: 19086-19098. CrossRef Medline

Holz MK, Blenis J (2005) Identification of S6 kinase 1 as a novel mammalian target of rapamycin (mTOR)-phosphorylating kinase. J Biol Chem 280: 26089-26093. CrossRef Medline

Houlden H, Baker M, Morris HR, MacDonald N, Pickering-Brown S, Adamson J, Lees AJ, Rossor MN, Quinn NP, Kertesz A, Khan MN, Hardy J, Lantos PL, St George-Hyslop P, Munoz DG, Mann D, Lang AE, Bergeron C, Bigio EH, Litvan I, Bhatia KP, Dickson D, Wood NW, Hutton M (2001) Corticobasal degeneration and progressive supranuclear palsy share a common tau haplotype. Neurology 56:1702-1706. CrossRef Medline

Jackson GR, Wiedau-Pazos M, Sang TK, Wagle N, Brown CA, Massachi S, Geschwind DH (2002) Human wild-type tau interacts with wingless pathway components and produces neurofibrillary pathology in Drosophila. Neuron 34:509-519. CrossRef Medline

Kabeya Y, Mizushima N, Ueno T, Yamamoto A, Kirisako T, Noda T, Kominami E, Ohsumi Y, Yoshimori T (2000) LC3, a mammalian homologue of yeast Apg8p, is localized in autophagosome membranes after processing. EMBO J 19:5720-5728. CrossRef Medline

Kim BM, You MH, Chen CH, Lee S, Hong Y, Hong Y, Kimchi A, Zhou XZ, Lee TH (2014) Death-associated protein kinase 1 has a critical role in aberrant tau protein regulation and function. Cell Death Dis 5:e1237. CrossRef Medline

Koch JC, Tönges L, Barski E, Michel U, Bähr M, Lingor P (2014) ROCK2 is a major regulator of axonal degeneration, neuronal death and axonal regeneration in the CNS. Cell Death Dis 5:e1225. CrossRef Medline

Kouri N, Whitwell JL, Josephs KA, Rademakers R, Dickson DW (2011) Corticobasal degeneration: a pathologically distinct $4 \mathrm{R}$ tauopathy. Nat Rev Neurol 7:263-272. CrossRef Medline

Krüger U, Wang Y, Kumar S, Mandelkow EM (2012) Autophagic degradation of tau in primary neurons and its enhancement by trehalose. Neurobiol Aging 33:2291-2305. CrossRef Medline

Liu WK, Le TV, Adamson J, Baker M, Cookson N, Hardy J, Hutton M, Yen SH, Dickson DW (2001) Relationship of the extended tau haplotype to tau biochemistry and neuropathology in progressive supranuclear palsy. Ann Neurol 50:494-502. CrossRef Medline

Miron M, Lasko P, Sonenberg N (2003) Signaling from Akt to FRAP/TOR targets both 4E-BP and S6K in Drosophila melanogaster. Mol Cell Biol 23:9117-9126. CrossRef Medline

Mizuno T, Amano M, Kaibuchi K, Nishida Y (1999) Identification and characterization of Drosophila homolog of Rho-kinase. Gene 238: 437-444. CrossRef Medline

Nakagawa O, Fujisawa K, Ishizaki T, Saito Y, Nakao K, Narumiya S (1996) ROCK-I and ROCK-II, two isoforms of Rho-associated coiled-coil forming protein serine/threonine kinase in mice. FEBS Lett 392:189-193. CrossRef Medline

Nezis IP, Simonsen A, Sagona AP, Finley K, Gaumer S, Contamine D, Rusten TE, Stenmark H, Brech A (2008) Ref(2)P, the Drosophila melanogaster homologue of mammalian p62, is required for the formation of protein aggregates in adult brain. J Cell Biol 180:1065-1071. CrossRef Medline

Shibuya M, Hirai S, Seto M, Satoh S, Ohtomo E (2005) Effects of fasudil in acute ischemic stroke: results of a prospective placebo-controlled doubleblind trial. J Neurol Sci 238:31-39. CrossRef Medline

Tang Z, Bereczki E, Zhang H, Wang S, Li C, Ji X, Branca RM, Lehtiö J, Guan Z, Filipcik P, Xu S, Winblad B, Pei JJ (2013) Mammalian target of rapamycin (mTor) mediates tau protein dyshomeostasis: implication for Alzheimer disease. J Biol Chem 288:15556-15570. CrossRef Medline

Togo T, Dickson DW (2002) Tau accumulation in astrocytes in progressive supranuclear palsy is a degenerative rather than a reactive process. Acta Neuropathol 104:398-402. Medline

Verhoef LG, Lindsten K, Masucci MG, Dantuma NP (2002) Aggregate formation inhibits proteasomal degradation of polyglutamine proteins. Hum Mol Genet 11:2689-2700. CrossRef Medline

Wang Y, Mandelkow E (2012) Degradation of tau protein by autophagy and proteasomal pathways. Biochem Soc Trans 40:644-652. CrossRef Medline 